\title{
Confronting Models With Data: The GEWEX Cloud Systems Study
}

\author{
David Randall 1, 2, Judith Curry ${ }^{3}$, Peter Duynkerke ${ }^{4}$, Steven Krueger ${ }^{6}$, \\ Mitchell Moncrieff ${ }^{7}$, Brian Ryan $^{8}$, David Starr ${ }^{9}$, Martin Miller ${ }^{10}$, \\ William Rossow ${ }^{11}$, George Tselioudis ${ }^{11}$, Bruce Wielicki ${ }^{12}$
}

Submitted to the Bulletin of the American Meteorological Society

April 2002

1. Corresponding author

2. Department of Atmospheric Science, Colorado State University, Fort Collins, Colorado 80523

3. Univ. of Colorado, Department of Aerospace Engineering Sciences, Campus Box 429, Boulder, CO 80309

4. Royal Netherlands Meteorological Institute, P.O. Box 201, 23730 De Bilt, The Netherlands

5. Deceased

6. Department of Meteorology,University of Utah, 135 South 1460 East, Salt Lake City, Utah 84112-0110 USA

7. National Center for Atmospheric Research, Box 3000, Boulder, Colorado 80307

8. CSIRO Division of Atmospheric Research, Private Bag 1, Mordialloc, Victoria 3195, Australia

9. NASA Goddard Space Fight Center Mail Code 913, Greenbelt, Maryland 20771

10. European Centre for Medium Range Weather Forecasts, Shinfield Park, Reading RG2 9AX, United Kingdom

11. NASA Goddard Institute for Space Studies, 2880 Broadway, New York, NY 10025 USA

12. NASA Langley Research Center, Hampton, VA 23681-0001 


\section{Abstract}

The GEWEX Cloud System Study (GCSS; GEWEX is the Global Energy and Water Cycle Experiment) was organized to promote development of improved parameterizations of cloud systems for use in climate and numerical weather prediction models, with an emphasis on the climate applications. The strategy of GCSS is to use two distinct kinds of models to analyze and understand observations of the behavior of several different types of clouds systems. Cloudsystem-resolving models (CSRMs) have high enough spatial and temporal resolutions to represent individual cloud elements, but cover a wide enough range of space and time scales to permit statistical analysis of simulated cloud systems. Results from CSRMs are compared with detailed observations, representing specific cases based on field experiments, and also with statistical composites obtained from satellite and meteorological analyses. Single-column models (SCMs) are the surgically extracted column physics of atmospheric general circulation models. SCMs are used to test cloud parameterizations in an un-coupled mode, by comparison with field data and statistical composites. In the original GCSS strategy, data is collected in various field programs and provided to the CSRM Community, which uses the data to "certify" the CSRMs as reliable tools for the simulation of particular cloud regimes, and then uses the CSRMs to develop parameterizations, which are provided to the GCM Community. We report here the results of a re-thinking of the scientific strategy of GCSS, which takes into account the practical issues that arise in confronting models with data. The main elements of the proposed new strategy are a more active role for the large-scale modeling community, and an explicit recognition of the importance of data integration. 


\section{Introduction}

The use of data to evaluate models is fundamental to science. Although ideally evaluations can be controlled and optimized in the laboratory, in most cases atmospheric scientists have to perform model-data intercomparisons by taking advantage of the uncontrolled opportunities that nature provides. A model-evaluation project is complicated in at least two distinct ways. The technical complexities are obvious and daunting: Data must be collected and analyzed, models must be developed and run, and the two sets of numbers must be brought into meaningful juxtaposition. This is hard enough. An additional and equally complex task, however, is to foster communication and fruitful interactions among the diverse scientific communities whose cooperation and combined expertise are needed in order to carry out the technical work.

The GEWEX ${ }^{1}$ Cloud System Study (GCSS) is a case in point. GCSS was organized in the early 1990s by K. Browning and colleagues (Browning et al., 1993, 1994). The challenges that arise as GCSS brings observations and models together are a microcosm of challenges that face all of Atmospheric Science. Over a period of years, GCSS has devised what we call the "GCSS Process:" a mode of operation that appears to optimize its scientific productivity. The GCSS Process was devised partly through trial and error and partly through introspection. The primary purpose of this article is to outline the key elements of the GCSS Process, which, we believe, have the potential be useful for many atmospheric science projects.

1. GEWEX is the Global Energy and Water Cycle Experiment. 
The mission of GCSS is to facilitate the development and testing of improved cloud parameterizations for climate and numerical weather prediction (NWP) models. GCSS deals with collections of clouds acting as systems, spanning a range of scales. Browning et al. $(1993,1994)$ envisioned that the development of improved cloud parameterizations could be aided by the use of cloud-system-resolving models (CSRMs). These are models with high enough spatial and temporal resolution to represent individual cloud elements, and covering a wide enough range of space and time scales to permit statistical analysis of simulated cloud systems. According to Browning et al., CSRMs can be used as experimental testbeds to develop understanding, to produce synthetic four-dimensional datasets, and to test parameterizations.

It is important to recognize that, despite their high computational cost, CSRMs do not simulate cloud systems from first principles. Although the cloud-scale and mesoscale dynamical processes, which must be parameterized in atmospheric general circulation models (AGCMs), are explicitly simulated in CSRMs on scales down to a kilometer or so in the horizontal and 100 $m$ or so in the vertical, the important microphysical, turbulent, and radiative processes are still parameterized. Because CSRMs explicitly represent mesoscale and microscale dynamical processes, the scientists engaged in CSRM-based research tend to be mesoscale and/or microscale dynamicists.

A second important element of GCSS research involves the use of single-column models (SCMs). As the name suggests, an SCM is essentially the column physics of an AGCM, considered in isolation from the rest of the model, i.e. an SCM is that which the GCSS Process 
aims to test and improve. The key utility of SCMs is that they can be used to make connections between GCMs and data collected in the field, thus facilitating observationally based evaluations of new and supposedly improved parameterizations, in isolation from the large-scale dynamical framework of a GCM. Over the past several years we have seen the creation of SCMs in most of the global modeling centers around the world, including both climate modeling centers and NWP centers. Scientists who work with SCMs tend to be members of the large-scale modeling community.

Both a CSRM and an SCM can be considered to represent a GCM grid column. To use these models, we must first measure the large-scale meteorological processes that are acting on a column of the atmosphere. We then use the CSRMs and SCMs to compute the cloud formation and radiative transfer processes inside the column. Finally, additional observations are used evaluate the results produced by the models. This strategy is illustrated in Fig. 1. Field data are used to drive the SCM and CSRM, and also to evaluate the model results. CSRMs compute some quantities that are very difficult to observe, such as the four-dimensional distributions of liquid water and ice. Although this simulated information is no substitute for real observations, because as mentioned above CSRMs contain parameterizations that introduce major uncertainties, CSRM results can, nevertheless, be judiciously compared with SCM results in order to diagnose problems with the latter. Finally, a parameterization tested in an SCM can be transferred directly to a three-dimensional GCM. Further discussion of SCMs, including their important limitations, is given by Randall et al. (1996). 
An important premise of the concept outlined above is that CSRMs give more realistic simulations than SCMs. This is to be expected because CSRMs explicitly represent many processes that SCMs can only incorporate in a statistical manner, through various closure assumptions. Nevertheless, as noted by Browning et al. (1994), it is important to confirm the expected superiority CSRM results relative to SCM results. GCSS has accomplished this, through various case studies.

An example is shown in Fig. 2. Measurements by the $\mathrm{ARM}^{1}$ millimeter cloud radar (MMCR) in Oklahoma provided observed profiles of hydrometeor (cloud plus precipitation) fraction. Fig. 2 shows that the CSRM cloud fraction profiles are in reasonable agreement with the observed profiles of hydrometeor fraction, while many of the SCM cioud fraction profiles are much larger. Fig. 3 compares the cloud fraction profiles for the entire 29 -day Case 3 period as observed by the MMCR, simulated by the UCLA-CSU ${ }^{2}$ CSRM, and simulated by the NCEP ${ }^{3}$ SCM (based on the NCEP global model). Even with a perfect model and 3-hour time averaging, we cannot expect perfect agreement of the simulated cloud fraction over the large-scale CSRM/ SCM domain (with a diameter of $300 \mathrm{~km}$ ) with the cloud fraction observed by the cloud radar (at a point). Nevertheless, the CSRM cloud fraction is in good agreement with the observations, except on the first day, and around the middle of the simulation when a clear period was observed. There are significant differences between the NCEP SCM and observed cloud fraction

1. ARM is the Atmospheric Measurements Program sponsored by the U.S. Department of Energy.

2. UCLA is the University of California at Los Angeles, and CSU is Colorado State University.

3. NCEP is the National Centers for Environmental Prediction, operated by the National Oceanic and Atmospheric Administration. 
profiles, most notably in the SCM's underestimate of cloud fraction at high levels. The NCEP SCM diagnoses stratiform cloud fraction according to the relative humidity, and the convective cloud fraction according to the intensity of the convection. The total cloud fraction equals the convective cloud fraction if present; otherwise, it equals the stratiform cloud fraction. The 3hourly averaged surface rainfall rates, liquid water paths, and precipitable water amounts from the CSRMs are in significantly better agreement with the observations than are the corresponding results from the SCMs.

GCSS began its work by carrying out what we call the GCSS Process Mark 1, which is schematically depicted in Fig. 4. The diagram refers to three communities of scientists, represented by the rectangular boxes; these are "data collection community" the "CSRM community", and the "GCM/SCM community." In order for GCSS to accomplish its goals, these three groups have to work together.

Such cooperation must be fostered and encouraged because of "cultural differences" among the communities, including differences in scientific background, interests, goals, and thought processes. These cultural differences make it difficult for the communities to interact, and this difficulty slows the progress of our science. We view GCSS as a "melting pot" for engendering such trans-cultural interactions.

The flow of information in the GCSS Process Mark 1 is indicated by the arrows in Fig. 4. Data is collected in various field programs and provided to the CSRM Community. The CSRM Community uses the data to "certify" the CSRMs as reliable tools for the simulation of particular 
cloud regime. The CSRM Community then uses its models to develop parameterizations, which are provided to the GCM/SCM Community.

As GCSS has evolved, we have become aware that the GCSS Process Mark 1 is seriously incomplete and somewhat unrealistic. The experiences that led us to this conclusion are outlined in Section 2. A revised approach is discussed in Section 3.

\section{Experiences with the GCSS Process Mark 1}

GCSS began with four Working Groups (WGs), each defined with respect to a particular cloud-system type:

- WG1, which deals with boundary-layer clouds including stratocumulus clouds and shallow cumulus clouds;

- WG2, which deals with cirrus clouds;

- WG3, which is focused on extratropical layer cloud systems; and

- WG4, which investigates precipitating deep convective cloud systems.

In 1999 , an additional WG was created:

- WG5, which deals with polar clouds, recognizing the importance of these clouds for the ice-albedo feedback. 
The scientific goals of the five WGs are listed in Table 1. Each of the WGs has been quite active. Their accomplishments were summarized in some detail by Randall et al. (2000), and are only briefly sketched here.

WG1 aims to improve physical parameterizations of clouds, other boundary layer processes, and their interactions. The primary approach of WG1 has been to compare observations of cloud-topped boundary layers with simulations produced using SCMs and Large Eddy Simulation (LES) models. Most of the leading groups modelling boundary layer clouds have participated in the WG1 workshops, which have been held on a quasi-annual basis. The WG has focused strongly on entrainment at the tops of stratocumulus clouds and on the sides and tops of shallow cumulus clouds. This focus is justified by the great importance of entrainment for the evolution of boundary-layer cloud systems (e.g., Bretherton et al., 1999 a, b). WG1 has enjoyed fruifful interactions with large-scale modeling centers. The most effective interactions to date have been with ECMWF and with the United Kingdom Meteorological Office (UKMO). These centers have conducted evaluations of parameterizations within the framework of the WG1 case studies (e.g. Lock 1998, 1999; Lock and McVean, 1999; Lock et al., 2000).

WG2 focuses on cirrus clouds. Several state-of-the-art GCMs now explicitly predict the occurrence and amount of ice in cirrus clouds. These parameterizations are difficult to test against the available data; for example, we currently lack global measurements of cloud ice content. The task of WG2 is to evaluate the validity and/or stimulate the improvement of such parameterizations through application and improvement of theory (models) and data. WG2 now 
involves the vast majority of research groups concerned with the details of modeling cirrus clouds, with active participation by large-scale modelers and also by key researchers concerned with measurements of cirrus clouds. Several workshops have been held, each with strong participation by dozens of scientists from around the world.

WG3 deals with midlatitude cloud systems, which are among the most extensive on Earth. The southern ocean is covered with multi-layer cloud systems spawned by relentless baroclinic activity, while the northern-hemisphere storm tracks produce the brightest cloud albedos anywhere. This type of cloud system is of tremendous importance to the Earth's radiation budget and to the hydrologic cycle. WG3 has made extensive use of regional or "limited-area" models (LAMs), which can represent the four-dimensional structure of an extratropical synoptic system. Also, WG3 has made extensive use of satellite data, including data from the International Satellite Cloud Climatology Experiment (ISCC). In these two ways, the approach of WG3 differs considerably from those of the other GCSS WGs. Through four major case studies, WG3 has demonstrated that the various participating models produce very different simulations of middle-level and high-level clouds. The diabatic effects of sublimation, melting and evaporation strongly influence prefrontal circulations. In particular, subliming cirrus can serve to trigger prefrontal descent that suppresses middle-level cloud. The models tend to produce the correct cloud types in strongly forced situations but not in weakly forced ones. The WG has concluded that parameterized fall speeds and evaporation processes are quite important and should be the focus of a future study, which may be performed in collaboration with WG2, 
WG4 deals with deep, precipitating convective cloud systems, which are active over large portions of the Tropics and also during the summer over the mid-latitude continents. These cloud systems produce globally significant precipitation, which is associated with convective heating of the troposphere, as well as large cloud radiative effects at the surface (solar) and at the top-ofatmosphere (solar and infrared). The goal of WG4 is to improve the parameterization of these cloud systems in GCMs through an improved physical understanding of cloud system processes. These processes include deep cumulus convection (convective updrafts and downdrafts and associated mesoscale circulations), shallow cumulus convection linked to the boundary layer, elevated or mid-level shallow moist convection, stratiform cloud microphysics (including the ice phase), the interaction of radiation with clouds, and boundary layer turbulence. WG4 has conducted extensive studies based on TOGA COARE data (e.g. Bechtold et al., 2000; Redelsperger et al., 2000), and is currently working with ARM data.

In January 1999, the GEWEX Scientific Steering Group approved the formation of GCSS Working Group 5, which deals with polar clouds. A GCSS focus on polar clouds is motivated by poor understanding of the physical processes at work in the polar cloudy boundary layer, poor simulations of polar cloud, radiation, and boundary layer processes by current GCMs, and the predicted Arctic amplification of greenhouse warming. Several features of the polar climate contribute to the difficulties in simulating the cloud and radiation environment by GCMs. These include cold temperatures and low humidities, organized in complex vertical structures including inversions; unusual cloud types such as diamond dust, persistent mixed phase clouds, thin multiple cloud layers, and convection from leads in sea ice; and the highly reflective and 
heterogeneous snow/ice surface. To improve GCM simulations of polar cloud processes, WG5 aims to improve the parameterizations of mixed-phase-cloud microphysics; radiative transfer through a cloudy atmosphere; the formation and dissipation of boundary layer clouds; and the turbuient processes at work in stable boundary layers, which are prevalent in the Arctic especially in winter. WG5 projects consist of case studies that can be used to evaluate LES models, CSRMs, radiative transfer models, and explicit microphysics models. In addition, some datasets of longer period (e.g. greater than 3 weeks) are assembled specifically to evaluate single-column and NWP models. WG5 is presently using data from the FIRE Arctic Clouds Experiment (Curry et al., 2000 ) and Surface Heat Budget of the Arctic Ocean (SHEBA; Uttal et al., 2000). A major data processing and integration effort is underway to prepare case studies for WG5 activities. Data for future case studies are expected to be provided by the Atmospheric Radiation Measurement (ARM) program, which in March 1998 began a long-term deployment of instrumentation at Barrow, Alaska (Stamnes et al., 1999). WG5 also plans to work with other groups that are planning field experiments in Antarctica.

Early GCSS meetings were dominated by mesoscale and microscale dynamicists; there was some but not much participation by the GCM/SCM Community. From the beginning, GCSS has worked hard to engage the GCM/SCM Community. As part of this effort, GCSS conducted a workshop in November 1998, which was hosted by the European Centre for Medium Range Weather Forecasts (ECMWF). The meeting brought together a diverse group of over a hundred scientists with strong common interests, who nevertheless rarely hold joint meetings. The participants included global modelers with an interest in cloud parameterization, mesoscale and 
microscale cloud modelers, radiative transfer specialists, and remote sensing specialists. The Workshop succeeded in producing a sense of communication among the various groups, and this was in fact one of its primary aims. A further primary aim was to entrain new participants into the various GCSS WGs. The Workshop included both invited and contributed lectures, poster papers, break-out groups for discussion of assigned topics, and, of course, a plan to produce this Proceedings. A summary of the Workshop has been published (World Climate Research Programme, 2000). Perhaps the most important practical benefit of the Workshop was the exposure of the global modeling, radiative transfer, and remote sensing communities to the parameterization-testing opportunities offered by the various GCSS WGs. Interactions initiated at the Workshop have now given rise to scientifically productive participation in the WGs. The Workshop was intended to initiate scientific interactions which would then become ongoing, facilitated at least in part through the GCSS WGs. Indications are that the Workshop succeeded in this way too. A follow-up Workshop is planned for May 2002, in Kananaskis, Canada.

\section{An assessment, and mid-course corrections}

Broadly speaking, a successful GCSS project has one (or, ideally, both) of two outcomes:

- A promising new cloud parameterization, developed and/or tested through the activities of a GCSS Working Group, is adopted for use in a climate model, or an NWP model, or a CSRM.

- Previously unknown or unexplained cloud processes are simulated using a CSRM, thus providing a pathway to scientific understanding. 
The GCSS Process Mark 1 has produced results of both types. Five vibrant WGs are hard at work, generating integrated datasets, and publishing the results of various case studies based on these datasets. SCMs are now being used at virtually all global modeling centers. In addition, GCSS has successfully facilitated the development, testing, and applications of some interesting and useful new parameterizations. The 1998 ECMWF Workshop brought about successful trans-cultural interactions among the various participating groups.

Nevertheless, GCSS has not yet fully achieved its ambitious goals, for several reasons:

- Despite years of effort, it continues to be necessary to work hard to attract a good showing of global modelers to attend the WG meetings and participate (with SCMs) in case studies; higher levels of participation are needed from the GCM community.

- Experience shows that it is necessary for the GCSS WGs to spend a substantial fraction of their energy data integration, which consists of producing observation-based datasets suitable for use with the CSRMs and SCMs. Data integration was not sufficiently recognized as a major activity in the 1994 Science Plan, which did, however, envision the "preparation of carefully assembled case study data sets" consisting of model output together with observations. We now appreciate that such datasets are themselves among our most important products, because they provide a comprehensive and internally consistent portraits of the processes at work in the atmosphere. 
- As a result of the studies performed by the GCSS WGs, it has become abundantly clear that "synthetic datasets" generated using CSRMs and LES models are not yet sufficiently reliable to be used as proxies for real data, except in carefully selected cases and even then only with the utmost caution.

- At first, GCSS did not adequately recognize the important role of satellite data in the GCSS Process.

- GCSS would benefit from much more participation on the part of the radiative transfer community. Cloud-radiation interactions must and will play a more prominent role in GCSS WG studies.

- With five WGs holding annual meetings, plus annual meetings of the GCSS Science Steering Group and the GEWEX Science Steering Group and the GEWEX Modeling and Prediction Programme, the community is finding it difficult to cope with the demands for travel and preparations for meeting activities. Our cup runneth over.

Analogous problems have been encountered elsewhere in the Atmospheric Sciences.

To address these issues, we have developed a revised version of the GCSS Process, designed as a "mid-course correction" to improve the scientific productivity of GCSS. The concept is shown in Fig. 5. The key differences from Fig. 4 are the red and blue items. In brief, the existence and key role of a data integration community are now acknowledged, the GCM/ 
SCM community now plays a more active role in the GCSS Process, and the importance of satellite data, as well as field data, is now explicitly recognized.

First, consider the data integration activity. Simulations of cloudiness produced with climate models are very difficult to evaluate using field-study observations alone, because of the limited spatial and temporal sampling of such field studies. Datasets with greater spatial and time coverage, as well as long-term statistical composites, are needed to facilitate the evaluation of climate models. Even after the data have been collected, a strategy is needed for making connections between the data and various models of interest. The raw data must be integrated in order to yield products that can be used to force models or to initialize models or to evaluate model results. One of the primary tasks of GCSS, from the very beginning, has been to produce such integrated datasets. The integrated data products are provided to both the CSRM group and the GCM/SCM group.

GCSS now addresses the need for such datasets through an explicit data integration activity, which is indicated in Fig. 5. Just as global modelers ask questions of and receive answers from the CSRM community, both the global modelers and the CSRM community ask questions of the data, i.e., they learn by directly confronting their model formulations and their model results with observations. In addition, modelers can uncover (and already have uncovered) problems with the observations. For example, modeling studies performed by GCSS WG4 were instrumental in the discovery and correction of problems with the TOGA COARE sonde data. 
Certain intrinsic characteristics of the modeling and observing communities make it difficult to bring models and data together. For the most part, observers are content to develop and (sometimes) apply retrieval algorithms to produce a data stream, and feel that their responsibility stops there. Climate modelers want neat, gridded, averaged, and in short "ready-toeat" geophysical variables presented as data products. They do not want to hear about or think about random errors or sampling biases. They cannot make use of raw radiometer data, or raw lidar data, or raw cloud radar data, or raw satellite data, or raw sonde data, or raw profiler data, or raw aircraft data. They lack the expertise to make meaningful use of raw data. Moreover, such the analysis of raw data is a full time job, which, if undertaken by the modelers, would preclude timely modeling progress. Data integration is the process by which we bridge this yawning gap between what the data-collection community provides and what the modeling community needs. Data integration consists of bringing together data from disparate instruments, and combining them into a coherent and comprehensive physical description of what was observed, in a form suitable for use in the evaluation of the relevant models.

To facilitate the efficient production of integrated datasets, we have created, within GCSS, a panel-based activity called "Data Integration for Model Evaluation (DIME)." DIME was formed to coordinate collection, analysis, and dissemination of integrated datasets for the case studies used to evaluate cloud system models and the parameterizations of clouds in GCMs. The tasks of DIME include:

- Coordination of data collection, quality checking, product definition, re-formatting, 
archival, and dissemination of a set of case study datasets.

- Generation of diagnostic datasets for each case study by combining "local" datasets from field campaigns that produced comprehensive sets of surface-based and aircraft observations with "global" satellite and re- analysis datasets.

- Provision of liaison between GCSS and GRP.

- Limited analysis and comparison of independent measurements to document measurement uncertainties.

- Collection of sets of cloud process model outputs for each case study to be combined with the observations in the final products.

- Development of a linked set of Web pages containing documentation, bibliographies, and links to additional related data sources.

Although GCSS at large has been carrying out these tasks at the Working Group level for some years now, DIME is now centralizing and coordinating the activity, thus eliminating unnecessary duplication of effort across Working Groups, and fostering the generation of more uniform integrated data products.

We now turn to the role, within GCSS, of the large-scale modeling community. A key goal of GCSS is is to foster the development of improved cloud parameterizations for use in 
climate models. Predictably, however, GCSS has to a large extent been distracted from true parameterization development and evaluation what we call the "Intercomparison Trap." Many (although not all) of the GCSS WG activities to date have involved organizing case studies, simulating the cases with multiple CSRMs and other models, and intercomparing the model results and the data. It is a matter of record that such intercomparisons can and sometimes do yield useful scientific results; an example is shown in Fig. 2, which definitely provides scientifically useful information. Intercomparisons are especially valuable for establishing community benchmarks and for exposing occasional gross errors in particular models. In the absence of active model development and other substantive scientific work, however, benchmarks would be of little value. For this reason, intercomparisons should be a "background" activity of GCSS, rather than its primary modus operandi. GCSS must focus primarily on specific scientific questions related to cloud parameterization, so that parameterization development occurs. We are therefore consciously steering our work away from the intercomparison mode, and focusing more on the focused evaluation of specific parameterizations used in climate models.

Note that we said "evaluation" rather than "development." No one should imagine that simply running CSRMs driven with data somehow leads to the development of new parameterizations. The CSRMs and SCMs are only tools for testing ideas. The solutions to our problems are the ideas themselves, which can be codified in the form of cloud parameterizations. The GCSS Process Mark 1, as summarized in Fig. 4, portrays the CSRM community as the primary producers of ideas in the form of parameterizations, and the GCM/SCM community as 
relatively passive consumers of these ideas. Experience shows that this is unrealistic, for two reasons. First, the CSRM community does not necessarily know what the GCM/SCM community wants or needs. Second, to the extent that the GCM/SCM community is viewed as playing a relatively passive role, it becomes difficult to involve them in the GCSS WG activities to the extent desired. In short, we have learned that parameterization development requires the active participation of large-scale modelers as well as cloud-system modelers.

The CSRM community has wonderful computational tools, but these tools must be focused on issues of relevance to the GCM community. This has in fact happened in some cases, especially in WGs 1 and 4. We note, however, that these two WGs have enjoyed a relatively high level of participation from the GCM community, and that in fact the GCM-oriented participants have already played a significant role in influencing the research conducted by WGs 1 and 4 .

In the GCSS Process Mark 2, the GCM/SCM community plays a key role by posing questions; this is indicated very explicitly in Fig. 5. The questions posed by the GCM/SCM community are closely associated with the conceptual underpinnings of the parameterizations proposed by the GCM/SCM community; for example, a question might be of the form "Is closure assumption X realistic?" Answers are provided by the CSRM community, based on their CSRM simulations and comparisons with observations. In short, GCSS now recognizes the need for the GCM/SCM community to play an active role in GCSS, and to provide the CSRM community with guidance concerning current issues in cloud parameterization as perceived by the GCM/SCM community. 
It order for this to work, GCSS must attract sufficiently many representatives of the global modeling community to GCSS WG meetings, and GCSS must ensure that the global modelers have an active and visible role in the activities. Here a simple practical strategy is proposed: Each GCSS WG meeting now features one or two presentations of specific new parameterizations and their performance in SCM tests (and other tests), to be presented by invited representatives of the GCM community. These presentations are designed to "pose questions" in the sense of Fig. 5.

Within the global modeling community there is a cadre of radiative transfer specialists. Radiative transfer is among the most climatologically important processes at work in cloud systems. GCSS must address the role of radiative transfer through cloud systems in order to achieve its goal of improving cloud system parameterizations for climate models. Some GCSS WGs have given an appropriate level of attention to radiative processes, while others have focused on cloud dynamical issues with prescribed radiative tendencies. It is essential that radiation processes receive a higher overall level of attention in future GCSS projects. In order for this to happen, it will be necessary to entrain radiative transfer specialists into the GCSS WG activities. The simplest and most effective way to do this is to proactively invite radiative transfer specialists into our WG meetings, give them an opportunity to present their science to the WGs, and engage in dialogs with the aim to identify scientific issues of mutual interest. This is an exercise in scientific match-making. The GCSS WG Chairs must take it upon themselves to bring the parties together, so that nature can take its course. 
SCMs and CSRMs cannot reveal the interactions of parameterized processes with the large-scale dynamics, simply because the large-scale dynamical processes are prescribed. This is an important limitation. The implication is that parameterizations must still be tested in full GCMs. The global modeling community includes the operational NWP centers as well as the climate modeling centers. Operational NWP provides excellent opportunities for comparing model results with data.

GCSS exists to provide and/or stimulate ideas and improvements in parameterization schemes used in both climate and NWP models. Nevertheless, the large-scale modelers continue to provide significant input to the GCSS by identifying the key problem areas for which existing parametrization schemes are inadequate (or non-existent), and which are considered crucial to the success of AGCMs.

NWP has a major role as the principal environment for developing and testing of schemes, and hence can provide feedback and focus to GCSS WGs. NWP can routinely compare the physics of its models with observations in the data assimilation and short-range forecasts environment. This allows the separation of problems specific to a physical process from the overall drift of longer climate-type integration.

Most.NWP centres now have an in-house SCM, based on their GCM, which serves as a testbed for the development and debugging of model parametrization codes. The SCMs are best utilized in parallel with the ability to extract column data from the forecast or analysis. The resulting data sets allow the timestep-by timestep sampling of the behavior and evolution of all 
parameters and the dynamical forcing at any location on the Globe. In the absence of forcing deduced entirely from observations (a difficult and inevitably limited task), the forcings extracted from the analyses or short-range forecasts allow a much greater range of situations to be studied.

Current short-and medium-range forecasts in NWP do not take into account variations in SST. This effectively disables many cloud feedbacks. Seasonal predictions, which are now being made operationally at various NWP centers, do include predicted sea surface temperatures and so cloud feedbacks on seasonal (and shorter) time scales can be examined in the context of seasonal forecasting.

\section{Concluding discussion}

GCSS is combining data with both high-resolution cloud-system models and singlecolumn models derived from lower-resolution global models, with the primary goal of developing and testing improved cloud parameterizations for use in the global models. As we have worked to carry out this program, have learned that the integration of observational datasets into "model-friendly" forms is an activity that must involve modelers in addition to the scientists involved in collecting the data. We now recognize such "data integration" as a major research activity that is essential for successful comparison of model results with data.

In addition, we have learned that parameterization development and testing is a process that can only succeed with the active participation of the global modeling community. For the most part, the global modelers know very well what their problems are, and what questions they need answers to. The observational community and the cloud-system modelers are positioned to 
provide answers to these questions. Dialogs are, therefore, just what we need. The GCSS Working Groups are organized so such dialogs occur frequently and naturally.

Recently, Grabowski and Smolarkwiewicz (1999), Grabowski (2001) and Khairoutdinov and Randall (2001) have begun exploring the use of CSRMs as "super-parameterizations," running interactively inside GCMs. This represents an intriguing new mode of interaction between the CSRM and GGM/SCM communities. We look forward to seeing its impact on the field. 


\section{References}

Bechtold, P., J.-L. Redelsperger, I. Beau, M. Blackburn, S. Brinkop, J.-Y. Grandpeix, A. Grant, D. Gregory, F. Guichard, C. Hoff and E. Ioannidou, 2000: A GCSS model intercomparison for a tropical squall line observed during TOGA-COARE. II: Intercomparison of singlecolumn models and a cloud-resolving model. Quart. J. Roy. Meteor. Soc., 126, 865-888.

Browning, K.A., A. Betts, P.R. Jonas, R. Kershaw, M. Manton, P. J. Mason, M. Miller, M.W. Moncrieff, H. Sundqvist, W.K. Tao, P.V. Hobbs, J. Mitchell, E. Raschke, R.E. Stewart, J. Simpson, 1993: The GEWEX Cloud System Study (GCSS). Bull. Amer. Meteor. Soc., 74, 387-399.

Browning, K. A., and collaborators, 1994: GEWEX Cloud System Study (GCSS) Science Plan. IGPO Publication Series No. 11,62 pages and 3 appendices, World Climate Research Programme, Geneva.

Curry, J. A., P. V. Hobbs, M. D. King, D. A. Randall, P. Minnis, G. A. Isaac, J. O. Pinto, T. Uttal, A. Bucholtz, D. G. Cripe, H. Gerber, C. W. Fairall, T. J. Garrett, J. Hudson, J. M. Intrieri, C. Jakob, T. Jensen, P. Lawson, D. Marcotte, L. Nguyen, P. Pilewskie, A. Rangno, D. Rodgers, K. B. Strawbridge, F. P. J. Valero, A. G. Williams, and D. Wylie, 2000: FIRE Arctic Clouds Experiment. Bull. Amer. Meteor. Soc., 81, 5- 29.

Grabowski, W. W., and P. K. Smolarkiewicz, 1999: CRCP: a cloud resolving convection 
parameterization for modeling the tropical convective atmosphere. Physica $D, 133,171$ 178.

Grabowski, W. W., 2001: Coupling cloud processes with the large-scale dynamics using the cloudresolving convection parameterization (CRCP). J. Atmos. Sci., 58, 978-997.

Khairoutinov, M. F., and D. A. Randall, 2001: A cloud resolving model as a cloud parameterization in the NCAR Community Climate System Model: Preliminary Results. Geoophys. Res. Lett., 28, 3617-3620.

Lock, A.P., 1998: The parametrization of entrainment in cloudy boundary layers. Quart. J. Roy. Met. Soc., 124, 2729-2753.

Lock, A.P., 1999: A parametrization of turbulent mixing in convective cloud-capped boundary layers derived from large-eddy simulations. In proceedings of GCSS-WGNE Workshop on "Cloud processes and cloud feedbacks in large-scale models", 9-13 November 1998, ECMWF, Shinfield Park, Reading, Berks., UK.

Lock, A. P. and M. K. MacVean., 1999: A parametrization of entrainment driven by surface heating and cloud-top cooling. Quart. J. Roy. Met. Soc., 125, 271-300.

Lock, A.P., A.R. Brown, M.R. Bush, G.M. Martin and R.N.B. Smith, 2000: A new boundary layer mixing scheme. Part I: Scheme description and single-column model tests. (submitted to 
Mon. Wea. Rev.).

Randall, D. A., K.-M. Xu, R. J. C. Somerville, and S. Iacobellis, 1996: Single-Column Models and Cloud Ensemble Models As Links Between Observations and Climate Models. J. Climate, 9, 1683-1697.

Randall, D. A., J. Curry, P. Duynkerke, S. Krueger, M. Miller, B. Ryan, D. Starr, W. Rossow, G. Tselioudis, and B. Wielicki, 2000: The second GEWEX Cloud Systems Study Science and Implementation plan. Int. GEWEX Project Office Ser. No. 34, 45 pp. Available from the International GEWEX Project Office.

Redelsperger, J.-L., P. R. A. Brown, F. Guichard, C. Hoff, M. Kawasima, S. Lang, T. Montmerle, K. Nakamura, K. Saito, C. Seman, W. K. Tao and L. J. Donner, 2000: A GCSS model intercomparison for a tropical squall line observed during TOGA-COARE. I: Cloudresolving models. Quart. J. Roy. Meteor. Soc., 126, 823-864.

Stamnes, K., Ellingson, R.G., J.A. Curry, J.E. Walsh, and B. D. Zak, Review of science issues and deployment strategies for the North Slope of Alaska/Adjacent Arctic Ocean (NSA/AAO) ARM site. J. Climate, 12, 46-63, 1999.

Uttal, T., J.A. Curry, M. McPhee, R. Moritz, D. Perovich, et al., 2001: Surface Heat Budget of the Arctic Ocean. Bull. Amer. Meteor. Soc., submitted. 
World Climate Research Programme, 2000: Workshop on cloud processes and cloud feedbacks in large-scale models. WCRP-110, WMMMO/TD No. 993, $175 \mathrm{pp}$. 


\section{Table Captions}

Table 1: Scientific objectives of the five GCSS Working Groups. 


Working Group 1 aims to improve physical parameterizations of boundary-layer
clouds. (http:/wwww.amath.washington.edu/ breth/GCSS/GCSS.html)
What controls the entrainment on the tops of stratocumulus, and on the tops and sides of
cumulus?

What are the physical processes that are responsible for the selection of cloud type (Sc versus

Cu) and cloud amount?

What are the consequences of cloud properties (micro- and macrophysical) on the cloud radiative properties and the energy balance at the Earth's surface and top of the atmosphere?

How are the mesoscale circulations in the cloud-topped boundary layer generated and how does the mesoscale variability interact with other processes such as: entrainment, radiation and drizzle?

Can the dynamics of the planetary boundary layer (PBL) be represented by a model that works across $\mathrm{PBL}$ regimes, or must different regimes be identified based on external criteria, and then modeled separately?

Working Group 2 focuses on cirrus clouds (http://eos913c.gsfc.nasa.gov/ gcss_wg2).

What level of microphysical complexity/sophistication is required for adequate treatment of cirrus clouds and their effects in large-scale models (climate and NWP)? A related critical question is: What level of microphysical complexity/sophistication is required for adequate treatment of cirrus clouds in remote sensing applications, both space-based and surface-based?

Table 1: Scientific objectives of the five GCSS Working Groups. 
What vertical resolution is required in large-scale models to enable adequate representation of the large-scale forcing to cirrus cloud formation?

To what extent is the parameterization of cloud dynamical processes and feedbacks (radiation latent heat - dynamics) required for the treatment of cirrus clouds in large-scale models? Similarly, to what extent must the ambient mesoscale (gravity) wave environment be explicitly taken into account?

What are the effects of the ambient aerosol population on cirrus cloud properties, and do variations in aerosols (or aerosol activation spectra via dynamics) lead to significant variations in cloud properties? How important is heterogeneous nucleation, and when is it important? Can/should the parameterization of cirrus clouds formed via large-scale ascent in a large-scale model be applied to cirrus formed via detrainment from deep convective cloud systems?

Working Group 3 focuses on midlatitude cloud systems (http:/hww.mscsmc.ec.gc.ca/GEWEX/GCSS/GCSS_wg3.html)

How important is it for AGCMs to realistically parameterize sub-grid scale mesoscale cloud structure and cloud layering in extra-tropical cloud systems?

What level of complexity of parameterized microphysical processes is needed in order that weather and climate general circulation models can realistically simulate extra-tropical cloud systems?

What is the validity of microphysical parameterizations in weather and climate general circulation models for mid-latitude cloud systems forced by orography?

Why are climate models deficient in developing clouds in the weakly forced regimes of midlatitude cloud systems?

Table 1: Scientific objectives of the five GCSS Working Groups. 
Why are the components of the water budget associated with mid-latitude cloud systems poorly represented in climate simulations?

WG4 deals with deep, precipitating convective cloud systems, which are active over large portions of the Tropics and also during the summer over the midlatitude continents (http://www.met.utah.edu/skrueger/gcss/wg4.html).

The occurrence (frequency and intensity) of deep convection. This includes the diurnal cycle of deep convection over land, and other interactions with the boundary layer.

The production of upper tropospheric stratiform clouds by deep convection. This includes the issue of microphysical complexity: how much is required in GCMs and NWP models?

Parameterized versus resolved motions as horizontal resolution increases. This is an issue now for mesoscale NWP models and for future global NWP models and GCMs.

Working Group 5 deals with polar clouds (http://paos.colorado.edu/faculty/ curry_home/index.html).

How does the phase of lower tropospheric clouds depend on temperature and aerosol characteristics, and how are mixed phase clouds maintained?

What is the mechanism that leads to the multiple-layering of cloud systems over the Arctic Ocean, and to what extent does this complex vertical cloud structure need to be resolved in GCMs?

To what extent must unusual features of the polar boundary layers (e.g. cloud top humidity inversions, heterogeneous underlying surface) be represented in GCMs to adequate simulate boundary layer clouds in the polar regions?

How do ciouds and their radiative effects influence the physical and optical properties of the snow/ice surface?

Table 1: Scientific objectives of the five GCSS Working Groups. 


\section{Figure Captions}

Fig. 1: Diagram illustrating how a CSRM and an SCM can be combined with field data to develop improved parameterizations for GCMs. The arrows in the figure show the "flow of information." This flow starts with the field data, in the lower right-hand corner of the figure. The observations collected during field are used with both the CSRM and the SCM, in essentially the same three ways for both models. First, both models are initialized from observations. Second, both are "driven" with the observations of, for example, large-scale vertical motion. Finally, the results that the two models produce, in response to this observed forcing, are compared against other observations collected in field, e.g. observations of cloudiness and surface radiation. Through data assimilation, field data also can be directly used by GCMs, although that is not part of the SCM approach. This figure is adapted from Randall et al. (1996).

Fig. 2: Comparison of simulated and observed cloud fractions, averaged over a three-week period at the Southern Great Plains site of the Atmospheric Radiation Measurements Program (ARM). The left panel shows SCM results, and the right panel shows CSRM results.

Fig. 3: Time-height cloud fraction for WG4 Case 3, surface to $16 \mathrm{~km}$ : (top panel) observed by MMCR (3-hour averages), (middle panel) simulated by UCLA-CSU CSRM (1-hour averages), and (bottom panel) simulated by NCEP SCM (3-hour averages). Color indicates cloud fraction, which ranges from 0 (violet) to 1 (red).

Fig. 4: The GCSS Process Mark 1, as envisioned by Browning et al. $(1993,1994)$. Data is collected and used to drive CSRMs. Analysis of the CSRM results leads to the development of 
improved cloud parameterizations, which are then provided to the large-scale modeling community. A revised version of this diagram is given in Fig. 5 .

Fig. 5: A revised GCSS Process; compare with Fig. 4. The key differences from Fig. 4 are indicated by the red and blue items in the present figure. Satellite data is recognized as having an importance comparable to that of field data. Data integration is now recognized as a key activity distinct from the others. The scientific questions that are posed in the process of parameterization development are now shown to originate within the GCM SCM community and/or the CSRM community. Answers to these questions are obtained through the use of CSRMs together with data. 


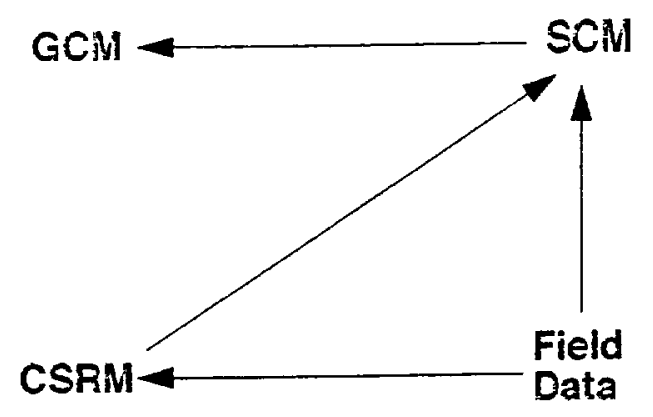

Figure 1

34 
CSRMs

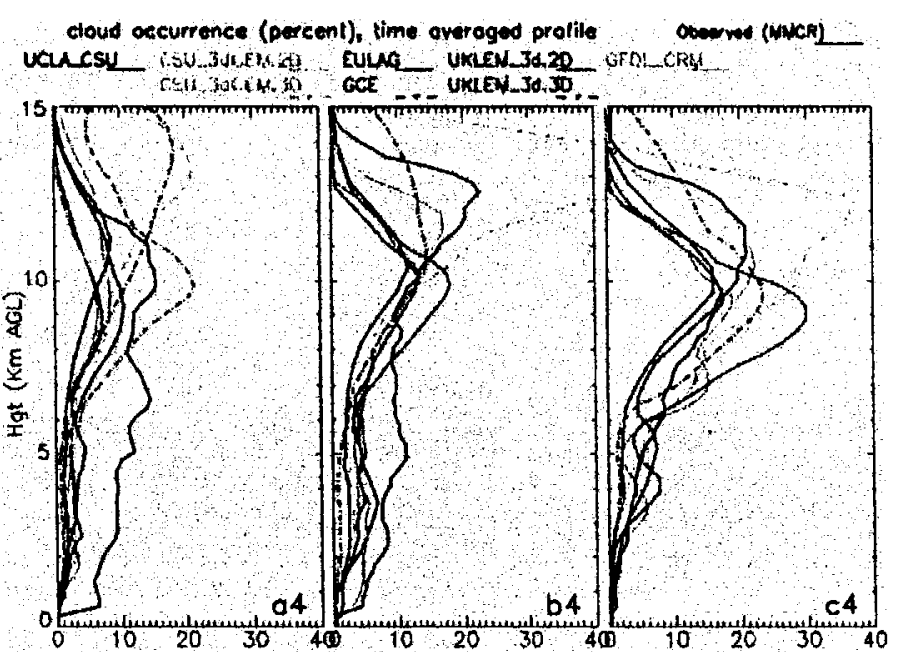

\section{SCMs}

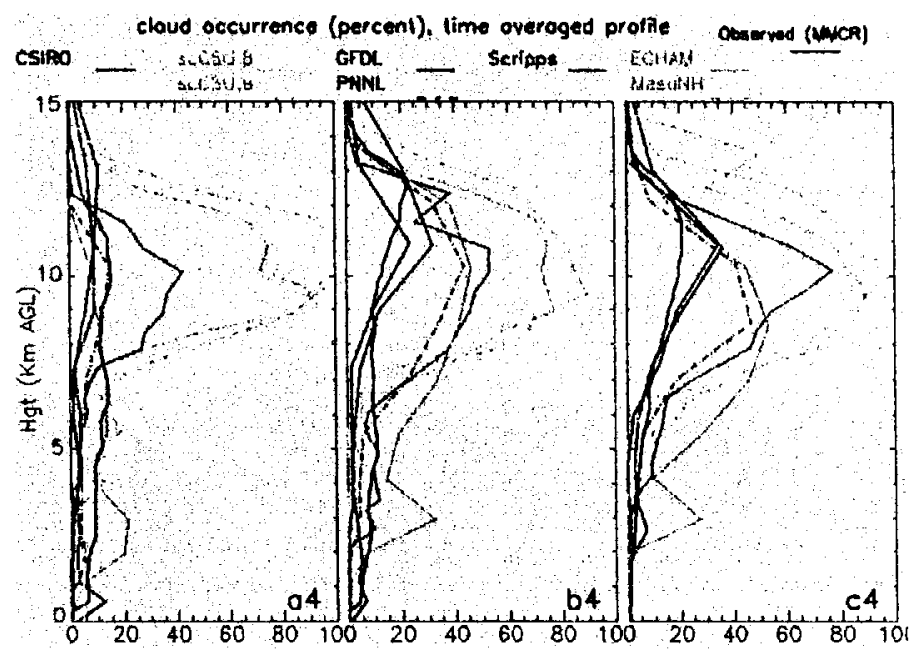

 


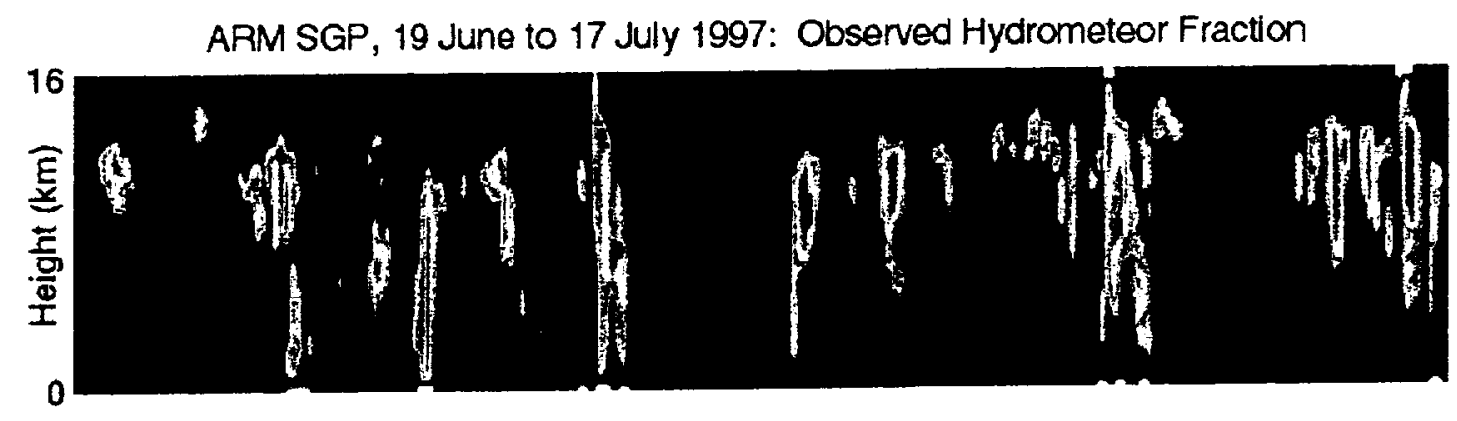

ARM SGP, 19 June to 17 July 1997: CSRM Cloud Fraction
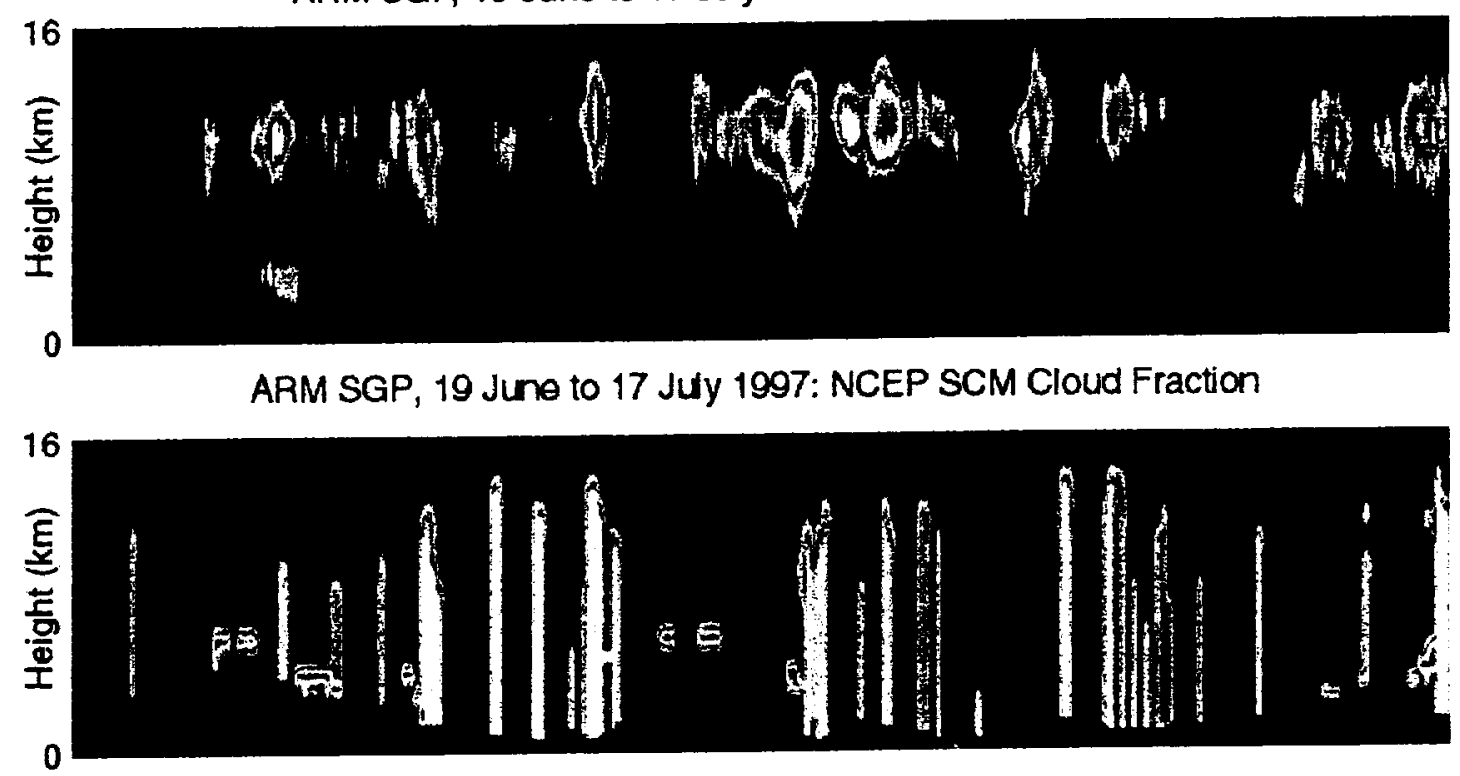

Figure 3 


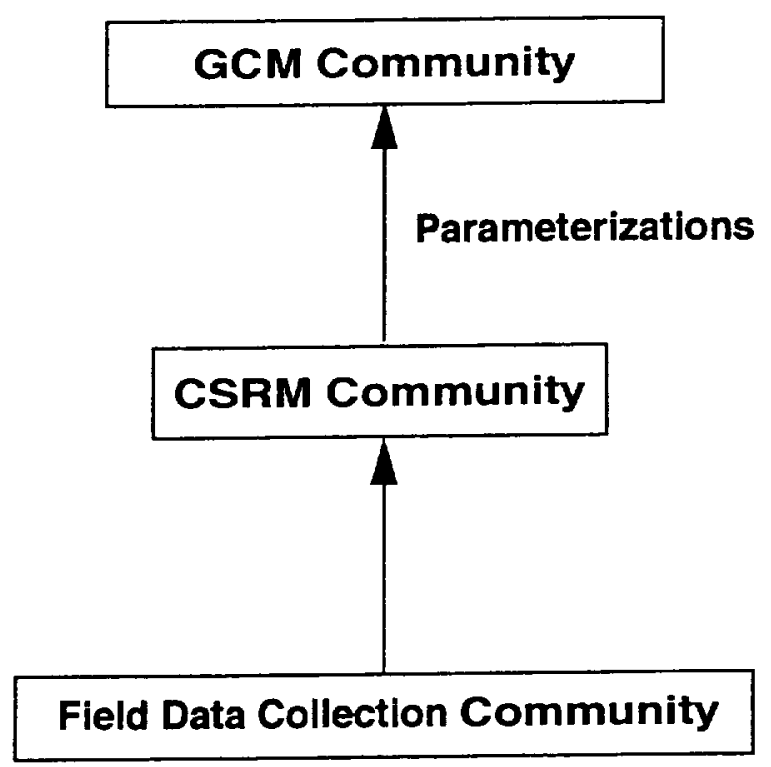

Figure 4 


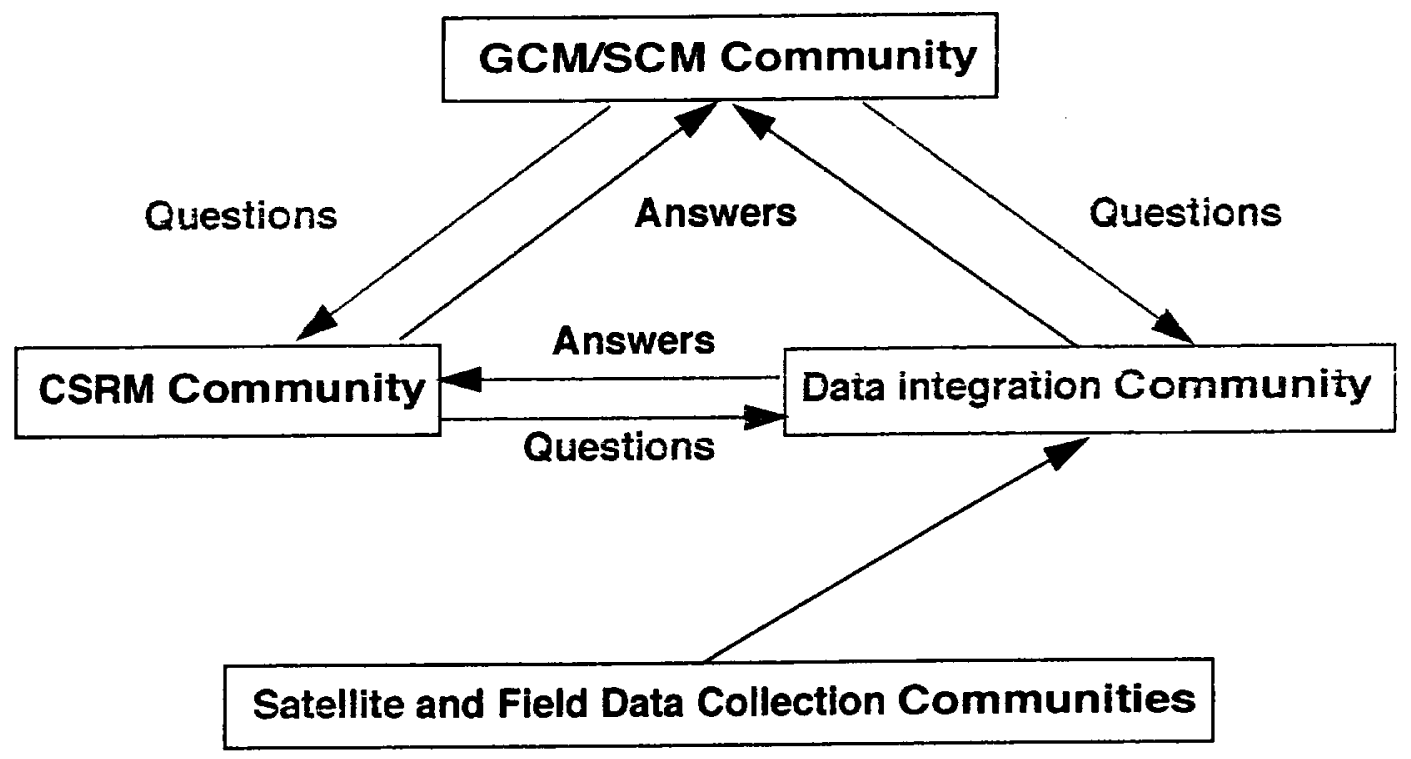

Figure 5 